\title{
Are You Realizing the Full Potential of Pharmacy Practice in the Healthcare System?
}

\author{
Lauza Saulnier
}

$\coprod_{\mathrm{p}}^{\mathrm{n}}$

today's complex and fast-paced healthcare environment, pharmacy leaders must ensure optimal utilization of resources for the delivery of efficient, safe, and high-quality services. High-performing pharmacy teams continuously strive to design and implement programs and services to improve patient health outcomes and to advance safe medication practices.

As a profession, though, have we maximized our contribution to the healthcare system? What progress have we made toward expanding the scope of practice? What strategies have we employed to prioritize practice activities that would have the greatest impact on patient care?

Monitoring of key performance indicators is one strategy that is required to assess the quality of clinical pharmacy practice and to support transparency and accountability. Unfortunately, such monitoring remains poorly integrated in many hospital pharmacy programs across the country. Several initiatives have been introduced in recent years to support evidence-informed medication management practices and to facilitate performance measurement.

The Canadian clinical pharmacy key performance indicators (cpKPIs) were developed, through a consensus process, to advance clinical pharmacy practice and thus to improve quality of care and patient outcomes (see https://cshp.ca/sites/ default/files/files/CSPH-Can-Concensus-cpKPI-KnowledgeMobilization-Guide.pdf). The core set of evidence-informed cpKPIs allows pharmacists working in acute care inpatient settings to concentrate their efforts on key clinical interventions; it also provides a structured approach to measuring the quality of direct patient care and delivery of services.

The Pharmacy Practice in Hospitals and Other Collaborative Healthcare Settings: Position Statements (available through https:// cshp.ca/position-statements) express the stance of the Canadian Society of Hospital Pharmacists (CSHP) and describe a desired and achievable level of performance that is applicable to the practice of pharmacy. This set of position statements serves as one building block for the CSHP's Excellence in Hospital Pharmacy program. The Excellence program is designed to assist members in focusing their efforts on improving patient health outcomes through patient-centred care, best practice, and communication and collaboration. Fifteen performance indicators have been chosen to measure progress toward excellence in pharmacy practice (https://www.cshp. ca/sites/default/files/ Excellence/Excellence Flyer_Revised.pdf)

For more than 30 years, the Hospital Pharmacy in Canada Report has been a leading reference and benchmarking tool for hospital

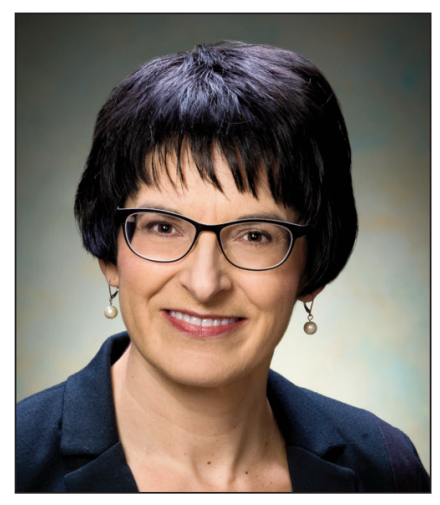
pharmacy services across Canada and around the world. In 2017, the CSHP Board accepted a request from the Hospital Pharmacy in Canada Editorial Board to become one of the Society's affiliated boards. The CSHP Hospital Pharmacy in Canada Survey Board, as it is now known, conducted its 2016/17 survey under the auspices of CSHP. The results were recently published at http:// hospitalpharmacysurvey.ca.

Performance measures published in the Hospital Pharmacy in Canada Report and those included in the Excellence in Hospital Pharmacy program help in monitoring progress toward objectives, comparing performance with benchmarks, assessing the real value of programs and services, and identifying improvement opportunities. Performance measures are crucial elements in driving change for the continuous pursuit of practice excellence.

CSHP has a long history of advancing pharmacy practice in hospitals and other collaborative healthcare settings. Let's challenge each other in achieving the best outcomes for our patients!

Lauza Saulnier, BSc(Pharm), ACPR, is Past President and Vision Liaison for the Canadian Society of Hospital Pharmacists. 